\title{
Intermolecular Binding of Functional Cyclic Compounds to RNA Nucleotides by an Ionic Process
}

\author{
Charles D. Schaper, Ph.D. \\ Transfer Devices, Inc. \\ Union City, CA 94587 USA \\ text: $510-378-8260$ \\ cschaper@transferdevices.com
}

\begin{abstract}
Exogenous RNA comprises the genetic material associated with several diseases which require immediate treatment, and thus mechanisms to hinder intracellular translation and reproduction of RNA viral agents are of great importance. Applying recent developments from this lab in methods relating to the interaction of DNA with steroid hormones, cyclic compounds are presented for intermolecular binding to nucleic acids. The requirements to achieve binding with RNA nucleotide pairs are described, which involve at a minimum functional elements positioned to interact with the lateral phosphate groups for each of the RNA strands through coupling with a positively charged ion, such as $\mathrm{Mg}^{2+}, \mathrm{Ca}^{2+}$, or $\mathrm{Zn}^{2+}$ ions; and an intermolecular hydrogen bond with the oxygen element of uracil at the carbon two location. Additional features of the binding molecules are examined for enhancements and differentiation in binding capability and include aromatic groups that have both a structural role of steric hindrance and a functional role to stabilize the binding mechanisms. Several categories of cyclic compounds are associated to have specific binding capabilities, and the interaction of these structures with potential receptor molecules are evaluated for assessment in delivery and binding of the compound to nucleic acids.
\end{abstract}




\section{Introduction}

As intracellular obligate parasites, positive sense and most negative sense RNA viral agents aim for replication within the cytoplasm $[1,2]$. Thus, endogenous protein structures of the host cell are inherent to the replication processes to infectious agents $[3,4,5]$. Consequently, in developing curative treatments, a reduction in the replication capability is sought in producing new drugs that attempt to hinder specific viral protein structures that do not have a human homolog [6]. Approaches that have been studied in developing curative treatments involve drugs that inhibit specific protein enzymes necessary for its translation [7]. Furthermore, there is the potential that solutions for one disease can be applied to other diseases in producing curative plans $[8,9,10]$ because of certain similarities in structure and function. Moreover, this approach of repurposing drugs, which already have been approved by the FDA, can enable significant savings in time and cost, and enhance safety $[11,12]$. However, it is unclear that the mechanism of action for one application is the same as that for another because of slight differences in the structural or functional characteristics of the enzyme targeted [11]. Furthermore, there are side effects that need to be examined in the repurposing of the drugs, which may limit the potency [13].

In this article, a treatment strategy, based upon specific structural configurations, is developed for the purpose of inhibiting the replication of RNA viral agents. The basis for these developments are recent discoveries made by this lab on the steroid molecule as the structural basis of DNA [14] and the symmetry of DNA nucleotides and steroid hormones $[14,15]$. These results involve the coupling, through an ionic binding process, of RNA nucleotides with designed cyclic compounds that can hinder the replication of the exogenous genomic content. The mechanism by which the binding takes place incorporates an intermolecular hydrogen bond, which is seen to be critical, to the uracil nucleotide, in combination with coupling of the molecule candidate to both strands of the RNA phosphate backbone, as enabled through ionic binding. In addition, the delivery vector is discussed because it is not enough for there to be a structural and functional match of the binding drug to the RNA nucleotide, as it is still necessary to deliver the binding cyclic compound to the RNA strands. Thus, with a detailed molecular analysis for binding onto RNA nucleotides, it is possible to develop effective treatment plans, and effectively enable a new class of antiviral agents, to hinder its progression.

\section{Results}

To initiate the development of strategies for interaction of cyclic compounds with RNA nucleotide pairs, an analysis of recent developments made by this lab on DNA nucleotides if first described, which will indicate the structural symmetry of cortisol, which is described in Section 2.1. After the discussion on DNA nucleotides, an analysis as described in Section 2.2 of the structure and functional arrangement of cyclic compounds that have structural symmetry with uracil and can bind onto the carbon 2 oxygen element of uracil and have 
a functional group at the carbon 5 position for binding onto the phosphate backbone. Then, compounds that have large cyclic structures, with electronegative functional groups positioned to interact with both sides of the phosphate backbone, and with a linear intramolecular structure are described in Section 2.3. A category of compounds that would be effective according to the mechanism is then presented in Section 2.4. The types of delivery vectors are developed in Section 2.5.

\subsection{Receptor Mediated Binding of Steroid Hormones to DNA Nucleotides}

As reference to the results on RNA, first critical results on DNA are presented. in a series of my preprints $[14,15]$, fundamental results were developed which indicated a very remarkable finding that showed for the first time that each of the base pairings of the four DNA nucleotides perfectly form the four ring structure of a steroid molecule, thereby indicating the structural basis of DNA. Furthermore, critical to a basic understanding of the functional nature of DNA and as will be seen to a basic understanding of a novel mechanism of action for the molecules described in this article, this finding led to a resolution of a basic mystery, stated as follows: why does the adenine-thymine pairing have only two internal hydrogen whereas cytosine-guanine have three internal hydrogen bonds. It was shown in my referenced preprints, and indicated in Figure 1, that the third hydrogen bond for A-T and T-A (and equivalently for RNA, Adenine-Uracil and Uracil-Adenine) is formed when the A-T and T-A nucleotides are coupled with corticosteroids, such as cortisol, which has an oxygen functional group that is perfectly positioned to form a hydrogen bond with the accessible oxygen-based functional group of thymine at the carbon two location. Apparently, this hydrogen bond is critical, as it can be evaluated in terms of comparing the activity of the synthetic corticosteroids prednisone and prednisolone [15], in which the only difference is the hydroxyl group at the mid-molecule location, but the activity of prednisone is absent, whereas prednisolone is five times more powerful than cortisol. Thus, the mid-molecule $\mathrm{OH}$ group enables the hydrogen bonding to thymine and results in activity. This bonding arrangement will be exploited to develop an entirely new class of therapeutics based on this new mechanism of action.

The structural relationship of this interaction of steroid hormone and DNA is presented in Figure 1(a), which uses the representative steroid hormones cortisol and testosterone in a manner such that each of the four nucleotide pairings are related to a steroid hormone to produce a set of three hydrogen bonds. Since the C-G and G-C pairing already have three internal hydrogen bonds, testosterone is paired; and since A-T and T-A only have two internal hydrogen bonds, cortisol is paired since in an amazing development the mid-section functional group of cortisol must couple with the unpaired oxygen element of Thymine at the carbon two position to form an intermolecular hydrogen bond. Moreover, it is noted that in order to get the structural match for each of the pairings, the T-A and C-G pairings were required for the hormone to be matched on the "back" of the molecule to its normal presentation. Effectively this forms a bijective structural code to DNA as expressed in 
Figure 1(b).

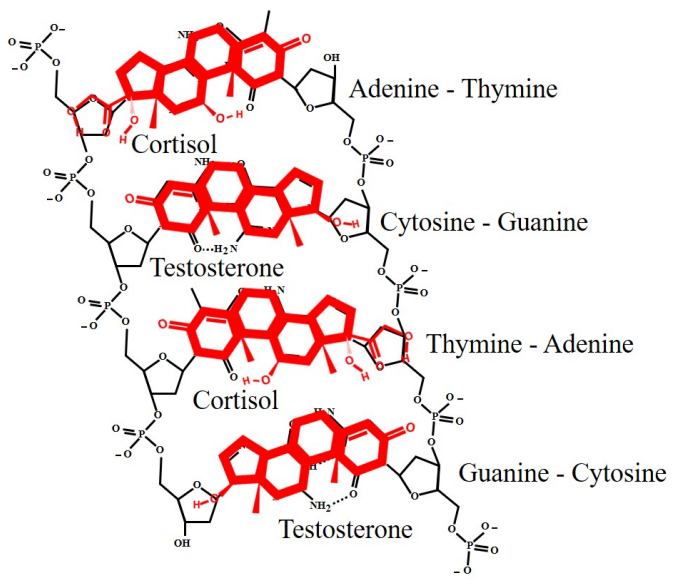

(a) Structural Symmetry

\begin{tabular}{|c|c|c|}
\hline $\begin{array}{c}\text { Base } \\
\text { Pair }\end{array}$ & $\begin{array}{c}\text { Steroid } \\
\text { Orientation }\end{array}$ & $\begin{array}{c}\text { Steroid } \\
\text { Class }\end{array}$ \\
\hline A-T & $\mathcal{F}$ & $\mathbb{H}$ \\
T-A & $\mathcal{B}$ & $\mathbb{H}$ \\
C-G & $\mathcal{B}$ & $\mathbb{S}$ \\
G-C & $\mathcal{F}$ & $\mathbb{S}$ \\
\hline
\end{tabular}

(b) New Code for DNA Nucleotides

Figure 1: As first described in my preprints [14, 15] and adapted from the recent preprint [16] for reference purposes, the structural basis of DNA is the steroid molecule, and that there is an exact correspondence to cortisol-like steroid hormone with the $A$-T and T-A pairs and the testosterone-like steroid hormones with the $C$ - $G$ and $G$-C pairs. This association produces three internal hydrogen bonds for each of the DNA nucleotide arrangements as the midmolecule $\mathrm{OH}$ group of cortisol-like molecules forms an intermolecular hydrogen bond with the unpaired oxygen of Thymine at the carbon two location. To achieve the configuration for each of the four pairs, as indicated in (a) there is a front-side and back-side orientation in order to get the three hydrogen bonds, and to have the proper orientation. (b) These developments thus produce a new code for DNA nucleotide sequences, which is comprised of the spatial relation of the steroid hormone to the DNA molecule on the front $\mathcal{F}$ or the back $\mathcal{B}$, and to the class of steroid hormone, either cortisol-like $\mathbb{H}$, with a mid-molecule functional group, or testosterone-like, $\mathbb{S}$. The structural match between DNA nucleotides and steroid hormones is perfect and undeniable.

While the structural match is critical, the functional purpose of the interaction also must be defined. I did that as well as first described in the preprints [14, 15]. To facilitate this binding process, it is necessary to first model the interaction of the steroid hormone with its receptor, because it will enable the alignment and transfer to the DNA structure. Here, the steroid hormone cortisol and its association with the glucocorticoid receptor is evaluated. It is noted that the conventional thinking on receptors of steroid hormones was that hydrogen bonding was only deployed, but I showed that the receptor is also capable of holding two ions that possess a charge of positive two each which can stabilize the complex and provide directionality. As shown in Figure 2(a), cortisol is arranged within representative residues of the ligand binding domain of the glucocorticoid receptor and two calcium ions are embedded [17]. In addition to stabilizing the complex, the ions provide an electrostatic potential with two separated positive charges that thereby provide alignment 
capability, as indicated in Figure 2(b). This stability and directionality enable the receptor complex to mobilize within the cell. This is an important result as the ion pair performs multiple functions, including stabilizing the receptor and the ligand, in this case the steroid hormone, and stabilizes the ligand after transfer to the DNA nucleotides.

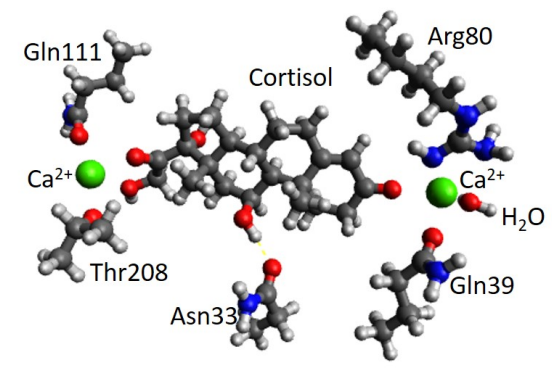

(a) Cortisol-Ca ${ }^{2+}-\mathrm{GR}$

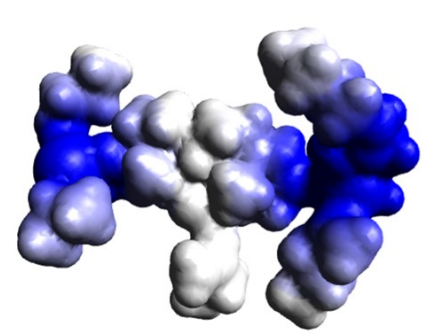

(b) Cortisol-Ca ${ }^{2+}$-GR electrostatic

Figure 2: In (a), the configuration of cortisol to the residues comprising the ligand binding domain of the glucocorticoid receptor indicating a combination of hydrogen bonding with coupling to calcium ions (shown in green); In (b), The electrostatic potential of the complex indicates two separate regions of positive charge separated by $16.3 \AA$. (Result and caption is adapted for reference from Figure 2 of preprint [16].)

After association with $\mathrm{Ca}^{2+}$ ions, the steroid hormone-receptor-ion complex interacts with the DNA molecule, which will later be applied within the context of RNA viruses. After passage into the $\mathrm{n}$, the steroid hormone- receptor-ion complex aligns through electrostatic interaction with the phosphate groups of adjacent and paired nucleotides of DNA. For cortisol, and according to Figure 1(a) and (b), it is also necessary that this alignment and interaction also involve the A-T or T-A pairing, since an available group of Thymine is required to achieve three internal hydrogen bonds. Upon transfer of the two ions and the steroid hormone to the DNA nucleotide pairs. Thus a stable structure results as indicated in Figure 3(a) in which the interaction of cortisol with $\mathrm{Ca}^{2+}$ ions and with the $\mathrm{PO}_{4}^{-}$ions of the A-T base pairing, thus forming a strong ionic bond. The calcium ions are stabilized at approximately the mid-way point of adjacent phosphate groups and the lateral separation. In Figure 3(b) the electrostatic potential map is indicated of the combined structure, and it is noted that there is a charge normalization in the middle with the retention of a smaller positive charge at the calcium ions. A significant stabilization of the DNA molecule results of $5,996 \mathrm{~kJ} / \mathrm{mol}$ [14], which can be distributed to induce gene transcription. However, for the analysis of RNA strands, and the coronavirus disease, this stabilization will be used to inhibit the RNA molecule from translation, as will be studied in the next section.

The calculations and developments for the steroid hormone-ion-DNA interaction is performed with the calcium ion as the coupling agent, but the results hold for other ions, particular $\mathrm{Mg}^{2+}$ and $\mathrm{Zn}^{2+}$. The point of the ion is to have significant positive charge to 


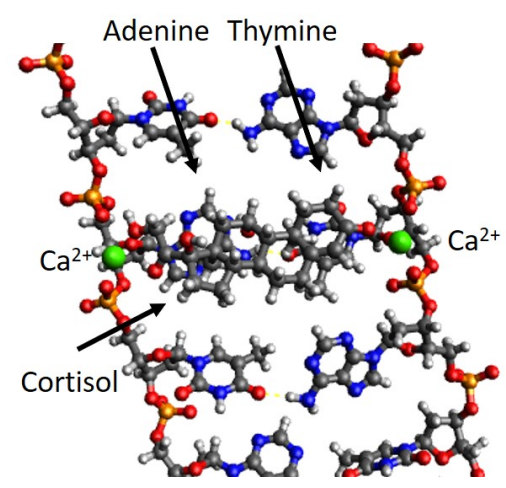

(a) Cortisol- $\mathrm{Ca}^{2+}$-Adenine-Thymine

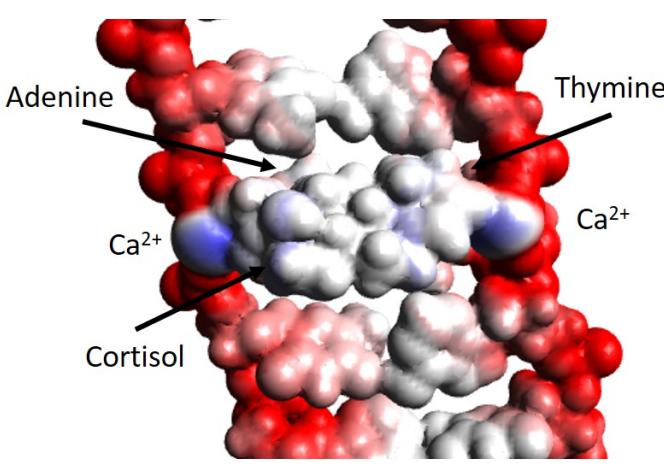

(b) Electrostatic Potential

Figure 3: As adapted from [16], in (a), after integration of the cortisol molecule with calcium ions, and release from the ligand binding domain, the interaction with the Adenine-Thymine pairing is shown, in which cortisol is also connected through intermolecular hydrogen bonding. It is noted that the calcium ions have stabilized at the middle of the adjacent phosphate groups (shown in orange). In (b) the electrostatic potential is indicated of the steroid-ion-DNA complex in which a small positive charge from the calcium ions is present. The distance between the $\mathrm{Ca}^{2+}$ ions is $1.66 \AA$, which is approximately equal to that corresponding to the ligand binding domain of Figure 2(b).

permit interaction with adjacent oxygen groups on the phosphate ions of the DNA or RNA nucleotides. Both $\mathrm{Mg}^{2+}$ and $\mathrm{Zn}^{2+}$ are utilized in our upcoming study on RNA to indicate the approach in a broader perspective for ionic elements and other ionic elements, such as $\mathrm{Mn}^{2+}$, that are capable of a positive two charge.

\subsection{Structural Symmetry with Uracil}

The first category assessed is the cyclic compounds with structural symmetry with Uracil, which includes a six-ring intramolecular structure that can pair with the oxygen group at the carbon two position, and with an electronegative element at the carbon 5 position. This structure can then tightly couple to Uracil, which is a key prerequisite to any drug that aims to bind with RNA nucleotides. The method by which the molecule aligns with adenine and the DNA phosphate backbone is then left to a subsequent cyclic chain of the compound.

For example, in Figure 4, a result is presented that indicates binding compatibility of the molecule dolutegravir with adenine-uracil nucleotide pair. There are several key connection, which includes: (1) the hydrogen bonding of the $\mathrm{NH}$ group of dolutegravir with the $\mathrm{O}$ element of Uracil at the carbon two position; (2) the oxygen element, incorporated within a cyclic structure, which aligns with the adjacent $\mathrm{PO}_{4}^{-}$lateral groups at the appropriate spacing; (3) the F element connected to the aromatic ring which will also align the RNA phosphate backbone. Also the aromatic ring will provide additional stabilization as a 
nucleophilic site which will allow the distribution of charge from uracil along with the $\mathrm{NH}$ group to promote attachment to uracil.

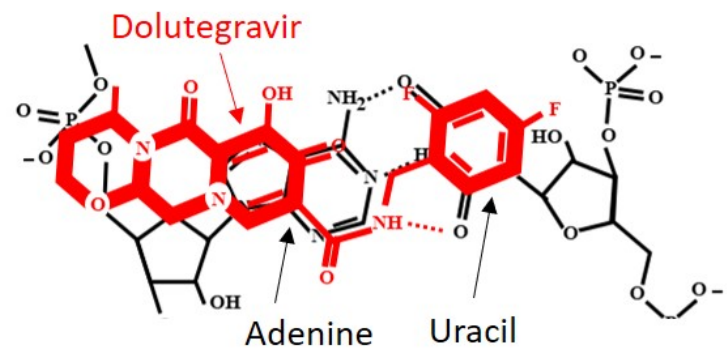

Figure 4: The chemical structure of dolutegravir indicates a strong match for binding with the Adenine-Uracil nucleotides. At either end there is a functional element perfectly positioned, with respect to an equivalency with hormones, that can associate a binding site based on ionic mediated interaction with the DNA phosphate backbone. Moreover, there is a functional NH group that can enable hydrogen bonding with the available functional group of Uracil, which apparently is a necessity for the binding to occur. The size is also a match and the aromatic ring will enable a strong binding, as well as an angle that is favorable for binding to RNA. The ring structures will increase the stability of the complex.)

The molecular model indicating the relationship of dolutegravir to the UA nucleotide pair is indicated in Figure 5(a) for a UAU feature. A calculation of the distances between the $\mathrm{Mg}^{2+}$ ions is $17.861 \AA$, and the distance between the functional end groups of dolutegravir is $14.452 \AA$, which indicates an average lateral distance to the ion pairs from the end elements of the molecule is $1.704 \AA$. The electrostatic potential of dolutegravir coupled to the adenine-uracil pair through the $\mathrm{Mg}^{2+}$ ionic elements, which indicates that there is positive charge differential at either end available for additional binding, and that the interior has neutral charge is indicated in Figure 5(b). In Figures 5(c) and (d) the coupling on the left side and right side of the pairs is indicated, which can also be measured indicating a tight relation. The distances are $1.779 \AA$ and $1.882 \AA$ to the phosphate oxygen elements, and $2.280 \AA$ to dolutegravir oxygen element on the left side, and on the right side, the coupling on the right side at the uracil nucleotide is indicated with the coupling agent positioned against the fluoride of dolutegravir, and the oxygen elements of the phosphate group. The distances are $1.723 \AA$ and $1.709 \AA$ to the oxygen elements, and $2.331 \AA$ to the fluoride element. There are several molecules that are similar to this structure and will be presented later in Section 2.4.

\subsection{Cyclic End Groups with Linear Intramolecular Structure}

The second category of compounds evaluate are those that have cyclic structures at each end which interacts with the RNA phosphate backbone, and has a linear internal struc- 


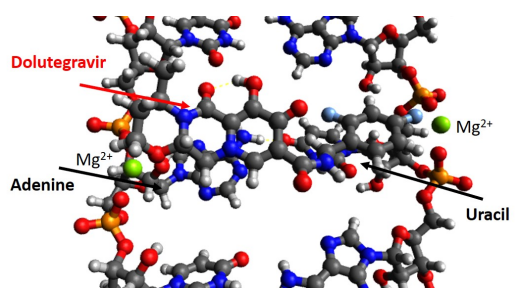

(a) Left Group End

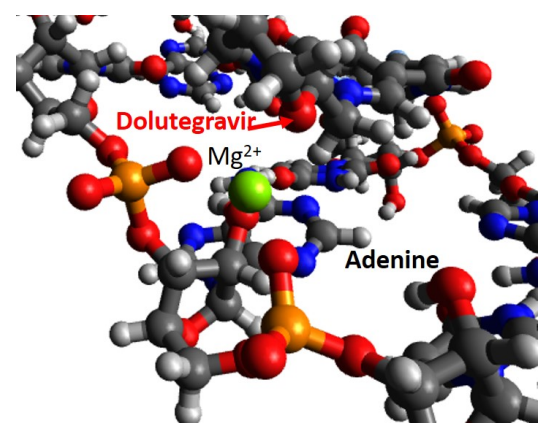

(c) Left Group End

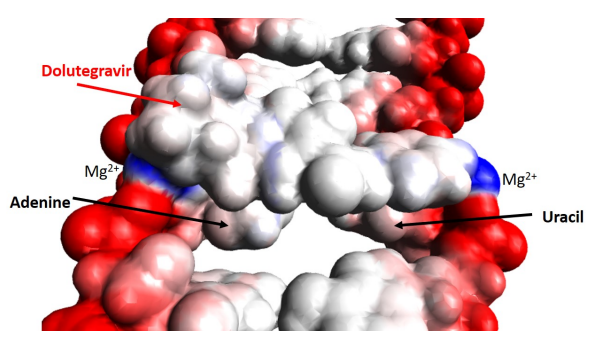

(b) Right Group End

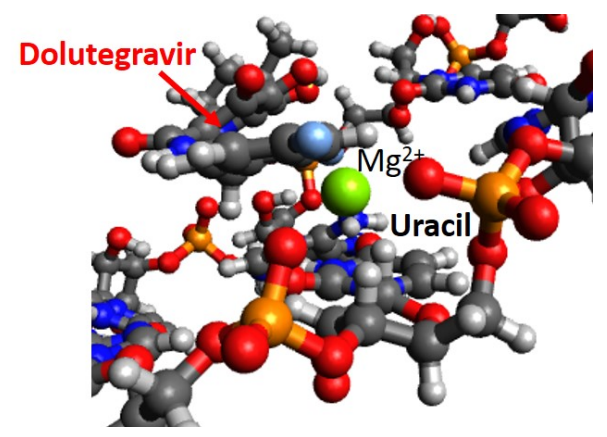

(d) Right Group End

Figure 5: (a) Molecular modeling to show the fit of dolutegravir within the RNA framework of the complementary nuclotide pair of adenine and uracil. The requisite size is of appropriate dimension and the interactions exist between the end groups and the DNA phosphate backbone. The distance between the ions is calculated as $17.861 \AA$, and the distance between the functional end groups of dolutegravir is $14.452 \AA$, indicating an average lateral distance to the ions to the $1.704 \AA$; In (b), the electrostatic potential of dolutegravir, as coupled to the adenine-uracil pair through the $\mathrm{Mg}^{2+}$ ionic elements, indicates that there is a positive charge differential at either end available for additional binding, and that the interior has neutral charge; In (c), the coupling on the left side of the adenine-uracil pair is indicated showing coordinated interaction of the oxygen elements of the phosphate groups, the ionic coupling agent, and the functional oxygen element in the outer ring of the dolutegravir molecule. The distances are calculated as $1.779 \AA$ and $1.882 \AA$ to the phosphate oxygen elements, and 2.280 $\AA$ to dolutegravir oxygen element. In (d), the coupling on the right side at the uracil nucleotide is indicated with the coupling agent positioned against the fluoride of dolutegravir, and the oxygen elements of the phosphate group. The distances are calculated as $1.723 \AA$ and $1.709 \AA$ to the oxygen elements, and 2.331 $A$ to the fluoride element.

ture that has functionality enabling its coupling with the uracil structure, although not necessarily in structural symmetry. This compound will have the capability to achieve strong binding at the ends but its internal structure will be weaker than that of the ringed structures, such as steroid structures. On the other hand, the compound due to its size 
will not fit within the glucocorticoid receptor, and thus will have greater efficacy and fewer side effects, at least from that perspective.

The structural and functional analysis of the molecules may comprise the category of protease inhibitors for nominal application, six of which are indicated in Figure 6, which indicates that these molecules have a certain structure which makes them well suited for binding to RNA nucleotides, and as will be shown for potentially binding and delivering $\mathbb{H}$ steroid molecules to RNA nucleotide pair of uracil-adenine. From the figure, each molecule has six sites that are structurally and functionally in common, which includes a hydroxyl group, amine group, and oxygen atom, as well as large structural ring groups, two on the end of the molecule, and one in the middle.
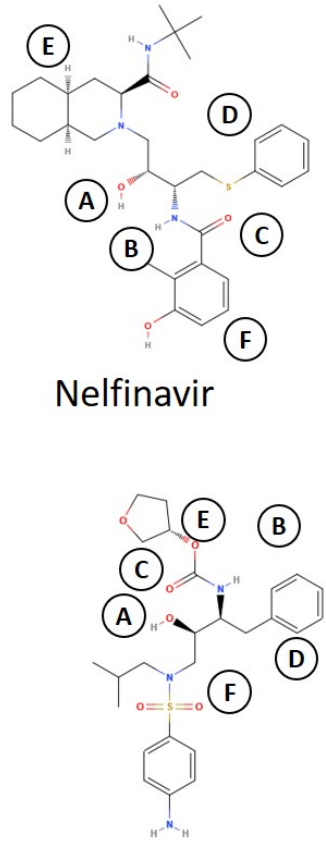

Amprenavir

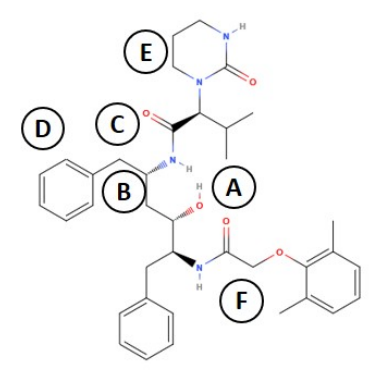

Lopinavir

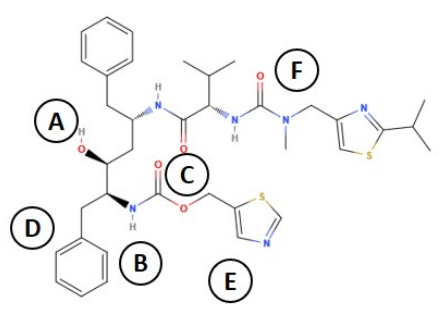

Ritonavir

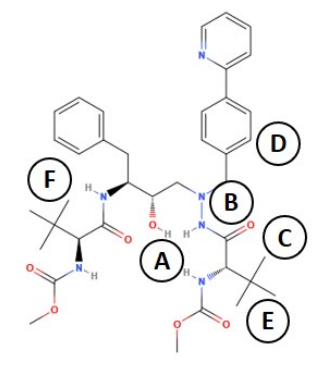

Atazanavir

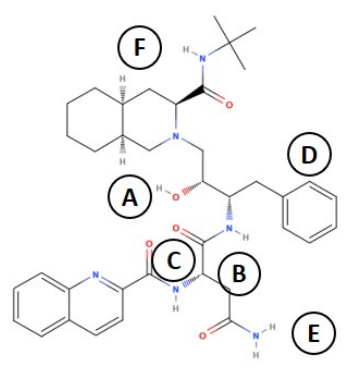

Saquinavir

Figure 6: For each of the molecules listed, there are common structural and functional characteristics that make them very well suited for interaction with RNA nucleotide pairs. A indicates a hydroxyl group in the central region of the molecule; $B$ indicates an NH group and $C$ an $O$ atom, both in proximity to $A ; D$ indicates a ring structure in proximity to $A$, $B$, and $C ; E$ and $F$ indicate large structures at either end of the molecule, both of which have nucleophilic characteristics.

The functional relationship, and structural characteristics, are described in Table 1. 
The $\mathrm{OH}$ and $\mathrm{NH}$ groups are important because it is capable of binding to the 2-carbon oxygen atom of Uracil. The rings provide steric hindrance, which importantly prevents the molecule from entering and binding to the active residues of the glucocorticoid receptor. This is important in order to minimize side effects, and to improve the availability and hence efficacy, of the molecule. It is critical for the molecule not to enter the glucocorticoid receptor, which will preclude the interaction of the receptor with cortisol, and will tie up the molecule such that it is unable to interact with the intracellular viral RNA. From a functional point of view, these large molecules, not only have a steric function, but also contain electrostatic potential of a nucleophilic nature, which will enable their interaction with the coupling ions, such as $\mathrm{Mg}^{2+}, \mathrm{Zn}^{2+}$, and $\mathrm{Ca}^{2+}$.

\begin{tabular}{|l|l|l|}
\hline Item & Unit & Direct Binding to RNA \\
\hline $\mathrm{A}$ & $\mathrm{OH}$ & Hydrogen Bond to Uracil \\
\hline $\mathrm{B}$ & $\mathrm{NH}$ & Hydrogen Bond to Uracil \\
\hline $\mathrm{C}$ & $\mathrm{O}$ & Coupling to Ion/PO $\mathrm{PO}_{4}^{-}$Group \\
\hline $\mathrm{D}$ & Ring & $\begin{array}{l}\text { Spacing/Alignment to RNA Nu- } \\
\text { cleotides; Steric hindrance to } \\
\text { Glucocorticoid Receptor }\end{array}$ \\
\hline E & Ring & $\begin{array}{l}\text { Nucleophile for First Ion/PO- } \\
\text { coupling; Steric hindrance to } \\
\text { Glucocorticoid Receptor }\end{array}$ \\
\hline F & Ring & $\begin{array}{l}\text { Nucleophile for Second Ion/PO } \\
\text { coupling; Steric hindrance to } \\
\text { Glucocorticoid Receptor }\end{array}$ \\
\hline
\end{tabular}

Table 1: The structural and/or functional characteristics of the category 2 molecules for interaction with the adenine to uracil nucleotide pairing. The central portion, the linear portion, of the molecule achieves a hydrogen bond with uracil, while the end groups are large, thereby enabling steric hindrance with entering the glucocorticoid receptor, while achieving stability when binding to the RNA phosphate backbone at either side.

To evaluate the effect, lopinavir is selected as a representative molecule, and is evaluated bind directly to double strand configurations of viral RNA. To develop these results, discoveries as described in my preprints $[14,15]$ as follows: (1) the structural analysis of nucleic acids that indicates steroid molecules as the basis and the relationship of DNA nucleotides to the structure of the steroid hormones that shows the corticosteroids binding to the uracil or thymine base, (2) the electrostatic driven ionic-phosphate-nucleotide coupling mechanism, which can be $\mathrm{Ca}^{2+}, \mathrm{Mg}^{2+}$, or $\mathrm{Zn}^{2+}$ ions for binding to the $\mathrm{PO}_{4}^{-}$groups of adjacent nucleotides; (3) the receptor mediated connection and molecular delivery mechanism of the corticosteroid to DNA nucleotides. Through molecular modeling, each atom and group comprising lopinavir can be defined, so as to deploy and design advancements effectively, ultimately to optimize the approach. 
In Figure 7, the binding of lopinavir to the RNA nucleotide pairing of uracil and adenine is indicated, starting with Figure 7(a), which shows that there are two nucleophilic ends capable of holding a positive ion of charge two, in this case $\mathrm{Mg}^{2+}$. The distance between the ions is $16.795 \AA$, which is consistent with the distance between the phosphate groups of the DNA and RNA nucleotides, as indicated in Figure 3(a) for cortisol to Adenine-Thymine pairing. The binding arrangement for Lopinavir to Uracil-Adenine is indicated in Figure 7(b), which shows that the two ends are coupled by magnesium to the RNA phosphate backbone. There is binding to the unmatched functional group of uracil, that is the oxygen atom on the carbon two of uracil (since the Adenine - Uracil coupling only has two internal hydrogen bonds, leaving one functional group of Uracil available). For this arrangement, since the Lopinavir molecule is a flexible chain, it allows tight hydrogen bonding to the NH group of $2.094 \AA$ to the carbon two oxygen atom of Uracil. For an alternative ionic coupling of $\mathrm{Zn}^{2+}$, the van der Waals hard shell is indicated in Figure $7(\mathrm{c})$, which shows the end groups in a tight binding configuration with the ionic coupling agent, for example $1.977 \AA$ and $2.108 \AA$ for the ion to phosphate oxygen distance, and $2.058 \AA$ for the ion to Lopinavir end group spacing.

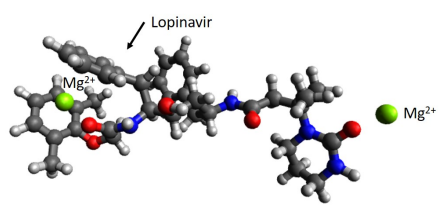

(a) Lopinavir- $\mathrm{Mg}^{2+}$

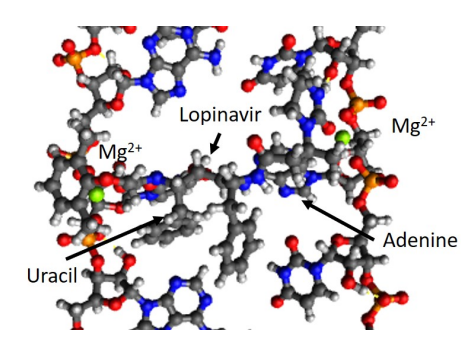

(b) Lopinavir- $\mathrm{Mg}^{2+}-\mathrm{UA}$ RNA

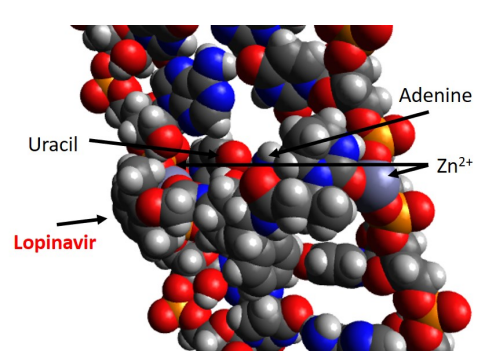

(c) Lopinavir- $\mathrm{Zn}^{2+}-\mathrm{UA}$ RNA

Figure 7: For binding Lopinavir to the RNA nucleotide pairing, (a) the configuration of lopinavir in relation to two $\mathrm{Mg}^{2+}$ ions (shown in green) indicating electrostatic attraction at the end groups; (b) The binding of lopinavir to the UA nucleotides including the binding with nonintramolecular-bound oxygen group of Uracil with an intermolecular bond to lopinavir; (c) The use of $\mathrm{Zn}^{2+}$ as the binding element instead of $\mathrm{Mg}^{2+}$, which show similar results.

Thus, with lopinavir alone acting without the aid of a receptor involves the intracellular diffusion of the molecule and coupling to ionic pairs such that binding can take place on the RNA strands that for a single-strand RNA agent are arranged in a secondary structure such that a stem configuration comprises paired RNA strands. By binding to the RNA double strand, the lopinavir stabilizes the overall molecule, and prevents its further processing, thus inhibiting its progression for some time prior to either its reactivation by loss of the binding, or in its degradation from interaction with the lysosome intracellular phagocytotic system. The double strand of RNA therefore is stabilized at multiple points, precluding its translation or introduction into the host genome. 


\subsection{Categories of Molecules in the Binding to RNA Nucleotides}

In Table 2, a total of four categories of cyclic compounds are presented, which are selected based upon characteristics, functional and structural, related to the potential interaction with the RNA nucleotide pairing of adenine and uracil. The class of steroid molecules were previously discussed and of that related to hydroxychloroquine have previously been demonstrated [16], and those of category 1 and 2 have been presented above. For category 1 molecules, the molecules show symmetry with the uracil nucleotide, both in structure and with binding to the carbon 2 oxygen, and of having a functional group at the carbon 5 position.

\begin{tabular}{|l|c|c|c|c|}
\hline Molecule & Uracil:O & Adenine:PO & Uracil:PO & Category \\
\hline Dolutegravir & $\mathrm{NH}$ & $\mathrm{O}$ & $\mathrm{F}$ & 1 \\
Cabotgravir & $\mathrm{NH}$ & $\mathrm{O}$ & $\mathrm{F}$ & 1 \\
Bictegravir & $\mathrm{NH}$ & $\mathrm{O}$ & $\mathrm{F}$ & 1 \\
\hline Lopinavir & $\mathrm{NH} / \mathrm{OH}$ & $\mathrm{O}$ & $\mathrm{O}$ & 2 \\
Nelfinavir & $\mathrm{NH} / \mathrm{OH}$ & $\mathrm{O} / \mathrm{OH}$ & $\mathrm{O}$ & 2 \\
Ritonavir & $\mathrm{NH} / \mathrm{OH}$ & $\mathrm{O}$ & $\mathrm{O}$ & 2 \\
\hline Hydrocortisone & $\mathrm{OH}$ & $\mathrm{O} / \mathrm{OH}$ & $\mathrm{O}$ & 3 \\
Dexamethasone & $\mathrm{OH}$ & $\mathrm{O} / \mathrm{OH}$ & $\mathrm{O}$ & 3 \\
Prednisolone & $\mathrm{OH}$ & $\mathrm{O} / \mathrm{OH}$ & $\mathrm{O}$ & 3 \\
\hline Hydroxychloroquine & $\mathrm{NH}$ & $\mathrm{OH}$ & $\mathrm{Cl}$ & 4 \\
\hline
\end{tabular}

Table 2: The three key binding sites are the two end groups associated with the RNA nucleotide pairing, in addition to the available oxygen element at the carbon two position of the uracil nucleotide. The types of molecules are separated into four categories as follows: the first category considers a symmetry with the uracil nucleotide, including hydrogen bonding at the carbon two position, and a functional group at the carbon five position capable of ionic coupling; the second category consists of large steric groups at the ends and in the center, along with a linear element connecting the two, capable of hydrogen bonding with the uracil nucleotide; the third category is that of the steroid hormones and molecules that are symmetric across adenine and uracil; the fourth category shows structural symmetry with the uracil, and a linear structure connecting to the adenine phosphate backbone.

From these relations, it is possible to optimize new molecules and design new compounds as drugs for binding directly onto the RNA nucleotides. As will be discussed in Section 2.5, the delivery mechanism also needs to be considered in the analysis. For example, while steroid hormones or molecules provide very strong binding to the RNA nucleotides, its delivery method should be considered, because its endogenous group, the glucocorticoid receptor, is not optimized for this purpose. Thus, a range of materials would seem to be an interesting method of analysis. 


\subsection{Delivery Vector}

In addition to binding onto the RNA nucleotides, it is necessary to determine the proper method of delivering the binding molecule for ionic coupling. There is a natural method for delivering onto DNA nucleotides for the purpose of gene transcription, but the mechanism to bind onto RNA nucleotides needs to be considered in the sense that the ultimate purpose is to stabilize the molecule such that it does not proceed with further processing. There is significant regulation in the delivery of steroid hormones to DNA nucleotides which involve large molecules to deliver the payload, but for RNA nucleotide, the mechanism needs to be developed towards simplicity and selectivity. Furthermore, dual purpose in which the delivery method can also be used as a binding molecule will improve the characteristics. The interaction with cortisol and the glucocorticoid receptor is also a key indicator to minimize side effects. In Figure 8, a representation of a delivery mechanism for Dolutegravir is indicated, in which the molecule is bound onto cortisol.

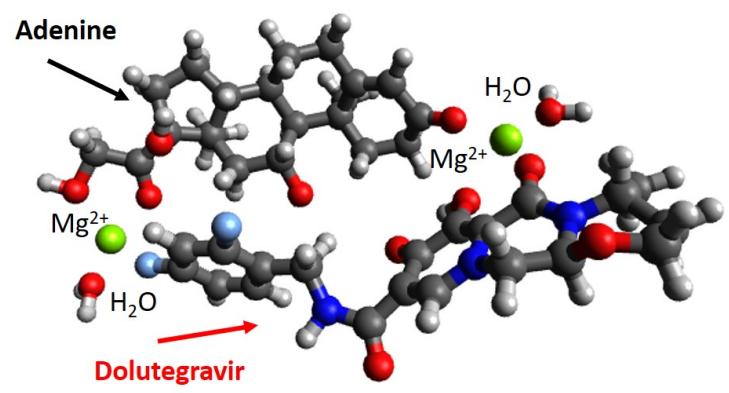

Figure 8: The delivery of dolutegravir and of cortisol to the RNA nucleotides are possibly achieved through a configuration centered about the two ions at each end, here shown for $\mathrm{Mg}^{2+}$, in which either molecule can attach to the adenine-uracil pairing, thereby increasing the rate of interaction.

In the development of a delivery mechanism, the manner similar to that with which steroid hormones interact with receptors is developed for the category of molecules whose structure is that of Category 2. As indicated in Table 3, the structure of these molecules are particularly compatible for functioning as a receptor for delivery of the corticosteroids for direct binding onto the RNA double strand, which can be formed also by secondary structures of single strand RNA. This would have the benefit that the corticosteroid molecules, which consist of multiple rings, would be a more stabilizing molecule than the direct binding of the linear molecule, with cyclic end groups, to the RNA strand, and thus would improve the efficacy of the RNA binding induced inhibition.

To illustrate the approach through molecular modeling, as shown in Figure 9(a), the Category 2 molecules are well suited for stabilizing and positioning the corticosteroid for binding with the RNA nucleotide, in this case exemplified by lopinavir and cortisol, coupled 


\begin{tabular}{|l|l|l|}
\hline Item & Unit & Delivery Vector for Steroid \\
\hline A & OH & Hydrogen Bond to Corticosteroid \\
\hline B & NH & Hydrogen Bond to Corticosteroid \\
\hline C & O & Hydrogen Bond to Corticosteroid \\
\hline D & Ring & $\begin{array}{l}\text { Steric hindrance to Glucocorti- } \\
\text { coid Receptor }\end{array}$ \\
\hline E & Ring & $\begin{array}{l}\text { Nucleophile for First Ionic Cou- } \\
\text { pling with Steroid; Steric hin- } \\
\text { drance to Glucocorticoid Recep- } \\
\text { tor }\end{array}$ \\
\hline F & Ring & $\begin{array}{l}\text { Nucleophile for Second Ionic } \\
\text { Coupling with Steroid; Steric } \\
\text { hindrance to Glucocorticoid Re- } \\
\text { ceptor }\end{array}$ \\
\hline
\end{tabular}

Table 3: The structural and/or functional characteristics of the category 2 molecules for delivery of corticosteroids to RNA nucleotides, as follows: $A, B$, and $C$ have molecular interactions associated with binding to the corresponding element of the corticosteroid. D has a purely steric hindrance role, while $E$ and $F$ have both a steric hindrance and molecular interaction roles.

with two $\mathrm{Mg}^{2+}$ ions. It is seen that the distance of the coupling ion elements is 13.829 $\AA$, which is smaller than the spacing between the target RNA nucleotides, indicating that the cortisol molecule is shaped, bowed outwards, with the methyl groups and the hydroxyl group facing and directed towards the uracil group. The alignment of the cortisol molecule and the absence of steric hindrance makes the lopinavir molecule effective for transporting cortisol to the adenine-uracil pair. It is also noted that there is intermolecular hydrogen bonding to the back of the cortisol molecule to the $\mathrm{NH}$ and $\mathrm{O}$ groups. Thus, the overall complex will be stabilized. An alternative orientation is evaluated in Figure 9(b), which flips the cortisol molecule such that the methyl groups and the hydroxyl group face towards lopinavir. Of course, this is not possible for an effective method to bind to RNA, and it does have a lack of intermolecular hydrogen bonding. The energy comparison of the two orientations is $-234 \mathrm{~kJ} / \mathrm{mol}$ which is less favorable than that of the preferred configuration with the methyl and hydroxyl groups facing outwards, which is $-313 \mathrm{~kJ} / \mathrm{mol}$.

The driving force to transition the cortisol molecule to the RNA strands is the electrostatic potential difference, and in Figure 10(a), the charge separation is indicated in which there are two concentrated areas of positive potential charge that is used to align the structure to the RNA strands. For single-strand RNA, there are secondary structures created due to the motion and looping of the strand inducing hydrogen bonding across complimentary base pairs arranged sequentially compatible. In Figure 10(b) the electrostatic potential map is indicated for an aligned association with the antiretroviral- 


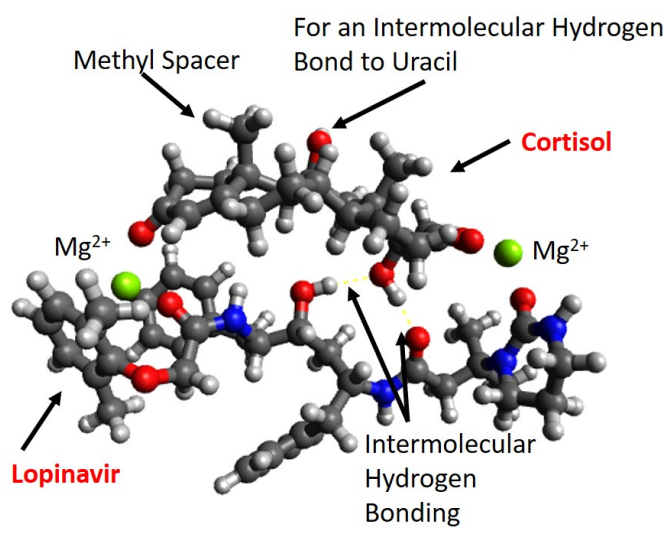

(a) Lopinavir- $\mathrm{Mg}^{2+}-$ Cortisol

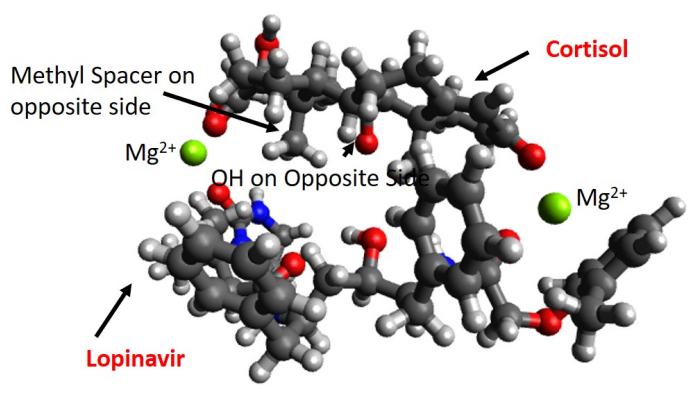

(b) Second Orientation

Figure 9: In the preferred orientation, (a) the association of Lopinavir, as a chemical delivery device, with Cortisol, held together by two ions. Note the intermolecular hydrogen bonding provided by the $\mathrm{OH}$ and $\mathrm{O}$ groups. The ends are both nucleophilic, for both cortisol and lopinavir, and are attracted to the two $\mathrm{Mg}^{2+}$ ions. Also, note that the methyl groups, and a hydroxy group is well positioned for interaction with Thymine. (a) In a second orientation, the cortisol molecule is flipped with the methyl groups facing towards lopinavir. However, there is a lack of hydrogen bonding. Thus, the energy level of this orientation is $-234 \mathrm{~kJ} / \mathrm{mol}$ which is less favorable than that of (a), which is $-313 \mathrm{~kJ} / \mathrm{mol}$.

ion-steroid complex to the RNA nucleotides configured as AUAUAUAU. The separated negative charge will attract the separated positive charge to achieve a binding complex.

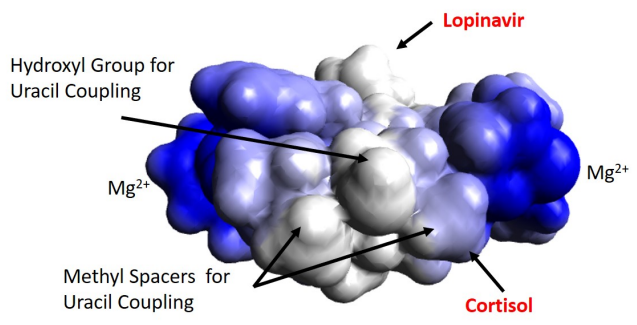

(a) Lopinavir- $\mathrm{Mg}^{2+}$-Cortisol Electrostatic

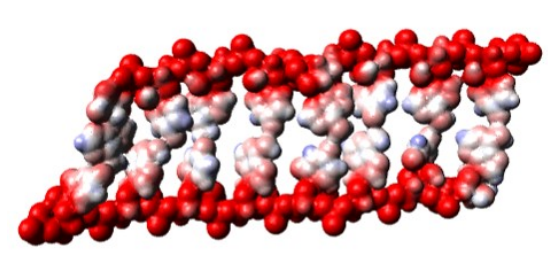

(b) Electrostatics of AUAUAUAU

Figure 10: In (a), the electrostatic potential of the Lopinavir- $\mathrm{Mg}^{2+}$-Cortisol complex indicating positive electrostatic potential, with two separated charges. The methyl groups and the hydroxyl group of cortisol are aligned and ready for interaction. In (b), the electrostatic charge potential, which is negative, is in a configuration compatible with the Lopinavir$\mathrm{Mg}^{2+}{ }_{-}$Cortisol complex, which will permit coupling.

In the initial presentation to the adenine-uracil nucleotide pairing, the cortisol molecule is positioned by alignment of the hydroxyl group to the corresponding oxygen element of 
the uracil base, achieving a hydrogen bond while the ion coupling lopinavir to cortisol begins to transition from the complex to the DNA phosphate backbone, as indicated in Figure 11(a). In completing the transfer of the ion element on either side of the nucleotide pairing, enabling a stable structure, which also includes an intermolecular hydrogen bond coupling. As shown in Figure 11(a), the ionic group is formed at the intersection of the neighboring oxygen groups associated with the phosphate group. The lopinavir transport molecule is free to diffuse away from the complex.

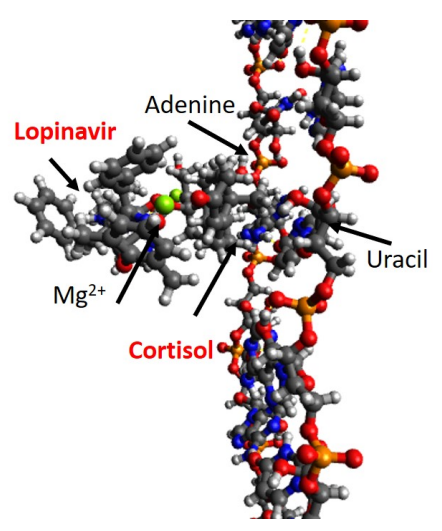

(a) Initial presentation

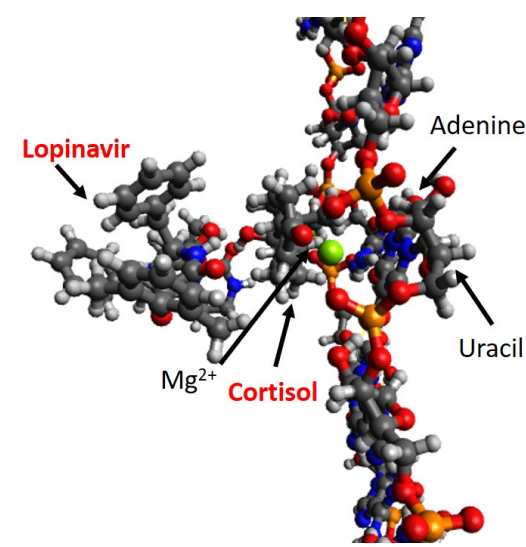

(b) Binding after ionic transfer

Figure 11: Side views: (a), the initial presentation of the lopinavir-Mg ${ }^{2+}$-cortisol complex to the AU nucleotides pair, driven by electrostatic potential alignment, which indicates that the orientation of the cortisol molecule is consistent with the uracil nucleotide in order to achieve an intermolecular coupling; In (b), after the transfer of the $\mathrm{Mg}^{2+}$ ion from the complex to the phosphate group on the backbone of the RNA strands, indicating a stable configuration.

The result of the final configuration is evaluated in Figure 12(a) and (b) in which the van der Waals hard sphere shells indicate tight coupling of the cortisol molecule to UA pair. The electrostatic interactions are indicated in Figure 12(c) and (d) showing the remainder of positive charge on each of the strands, which can participate in further actions. This could include the promotion of the removal of the binding complex through intracellular phagocytic mechanisms.

Thus, the pathway for the inhibition of the RNA viruses is straightforward, which involves the binding of the steroid molecule to the Category 2 compound, which functions as a corticosteroid molecular delivery device. After association of the complex, which includes two ions, it mobilizes in the intracellular space, and targets RNA double strands for binding, which occurs in a single transition process for both sides of the molecule and RNA nucleotides. After binding, the molecular delivery device is free to re-enage and ultimately exit the cellular space. 


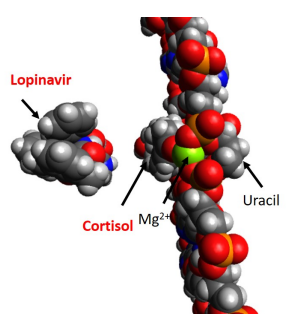

(a) End Group

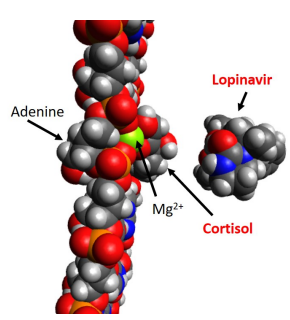

(b) End Group

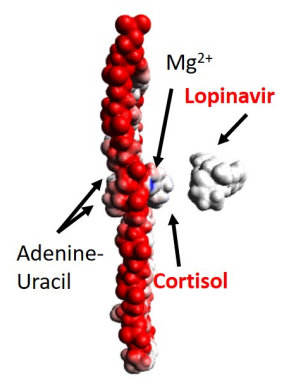

(c) Electrostatics

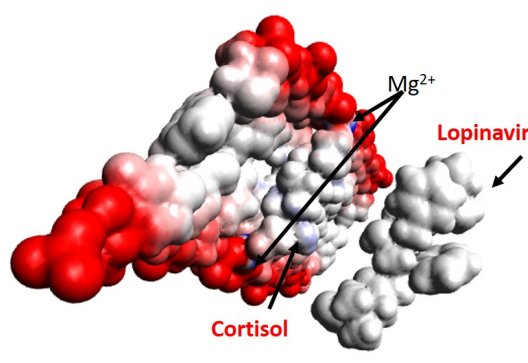

(d) Overall Electrostatics

Figure 12: In (a), the binding of the cortisol molecule to the RNA nucleotide is presented using van der Waals hard sphere, with lopinavir leaving, indicating a strong coupling of the steroid molecule to the RNA nucleotides. In (b), the binding of the steroid molecule on the other end group is presented indicating similar results. In (c), the electrostatic potential is indicated giving a size perspective of the coupling, and the neutrality of the lopinavir molecule. In (d), the coupling is presented at an angle to give a sense on the lateral coupling and the remainder of a positive charge for further processing.

\section{Discussion}

It is intriguing that there is a rich library of cyclic compounds, which are safe as evident by their approval for use by the FDA, that can be repurposed for application in binding to RNA nucleotides. As the source of many disease is exogenous RNA that originates by entry into the cytoplasm, the mechanism of an ionic binding process to RNA nucleotides thus enables a number of compound choices to potentially impede the progression of translation and replication. The particular selection can be determined by taking into account the potential side effects, which will have already been determined through their FDA analysis, and thus the risk can be lowered in the development of a plan.

To design a compound, the minimum requirements for binding to adenine-uracil RNA nucleotides, based upon the described models, are: (1) functional end elements that are well positioned for ionic coupling agent to the phosphate backbones; (2) a functional group that can interact though hydrogen bonding with the available functional group at the carbon two site of uracil. There are other criteria that can be used also to determine the efficacy of the approach and include the availability of surrounding functional regions to the uracil binding element which can enhance the interactivity, such as an aromatic ring, or at least a double bond positioned nearby. The rigidity of the binding molecule, for example determined by the number of its cyclic structures, is also important in stabilizing the overall complex, and thus hindering the ability of the RNA molecule to replicate. A representative compound that meets these criteria is the synthetic steroid prednisolone, which has a functional group 
positioned for interaction with uracil, in comparison to prednisone which instead has an $\mathrm{O}$ element, instead of an $\mathrm{OH}$ group, and thus does not form a direct hydrogen bond with uracil, and is therefore inactive.

While this research focused upon the adenine-uracil RNA nucleotides because of the available oxygen functional element at the carbon two position of uracil, and thus it was seen that there are a number of candidate molecules that could be captured because of this relation, there is the consideration that the analysis could be extended to the cytosineguanine pairing of the RNA nucleotides. In this instance, the coupling is possible through the RNA phosphate backbone, but would lack the intermolecular binding at the mid section of the complementary base pair, which would thus result in a loss of stabilization relative to adenine-uracil coupling. It would be necessary therefore to incorporate multiple links positioned near to one another in a manner similar to that described in [15], and possibly on opposite sides of the RNA nucleotide strands to offer an equivalent stabilizing force.

Since the structural and functional relations of the binding compounds described for RNA nucleotides will also apply to DNA nucleotides, it is useful to evaluate aspects that can be implemented to increase the likelihood for binding onto the RNA nucleotides only to improve selectivity. One area for differentiation is the delivery vector for the binding ion to associate the binding cyclic compound with the RNA nucleotides, which includes the interaction of the binding molecule with endogenous vectors, particularly the glucocorticoid receptor. Examples were presented on interaction of the binding molecule, and the delivery vector that can be designed such that it maintains occupancy within and exclusive to the cytoplasm without entry into the nucleus. For example, there is the potential for similar gene regulatory proteins to bind to inhibitory cytosolic proteins that can either anchor to cytosolic skeletal features or mask to nuclear localization signals to inhibit the interaction with nuclear import receptors.

The mechanisms described in this article of binding cycllic compounds to RNA nucleotides, which thus stabilize and inhibit the further progression of the RNA translation and replication process, can be designed and opimized for novel antiviral strategies.

\section{Methods}

To construct three dimensional molecular models to evaluate the interaction of antiretrovirals and corticosteroids with DNA and RNA nucleotides, and in combination with ionic coupling agents, the software program Avogadro was used. The amino acid residues of the ligand binding domain of the glucocorticoid receptor and the $\alpha$ carbon were positioned in proximity to the functional groups of cortisol in accordance with the approximate layout presented in [18]. This work involving the methodology of placing the cortisol molecule, along with ions using previously described methods [17]. The force field MMFF94 [19] was utilized and an optimization program was used by minimizing the energy associated with the complex so as to determine the bond length and the positions of the chemical elements, 
which also included the functional groups of the ligand binding domain. The dimensions of the spacings were determined by measurement at the central location of the elements: carbon, oxygen, hydrogen, nitrogen, calcium, magnesium, zinc, or phosphate. Using several starting points, the software optimization determined the optimal bond lengths and the respective positioning of the elements. Calcium, magnesium, or zinc ions with a positive two charge were situated in the vicinity of the negative electrostatic potential and the Avogadro software program was run to determine the optimal position of the ions. After determining the position, the molecule of interest and the two associated ions were extracted from the respective receptor and placed in the vicinity of the Adenine - Uracil pairing, and the software program was used to determine the optimal locations to minimize the overall energy, completing the simulation after convergence was achieved.

\section{Bibliography}

[1] E. De Clercq and G. Li, "Approved antiviral drugs over the past 50 years," Clinical microbiology reviews, vol. 29, no. 3, pp. 695-747, 2016.

[2] J. A. Hiscox, "Rna viruses: hijacking the dynamic nucleolus," Nature Reviews Microbiology, vol. 5, no. 2, pp. 119-127, 2007.

[3] T. A. Cooper, L. Wan, and G. Dreyfuss, "Rna and disease," Cell, vol. 136, no. 4, pp. 777-793, 2009.

[4] Y.-Z. Zhang, W.-C. Wu, M. Shi, and E. C. Holmes, "The diversity, evolution and origins of vertebrate rna viruses," Current opinion in virology, vol. 31, pp. 9-16, 2018.

[5] P. Poltronieri, B. Sun, and M. Mallardo, "Rna viruses: Rna roles in pathogenesis, coreplication and viral load," Current genomics, vol. 16, no. 5, pp. 327-335, 2015.

[6] J. L. Blanco, J. Ambrosioni, F. Garcia, E. Martinez, A. Soriano, J. Mallolas, and J. M. Miro, "Covid-19 in patients with hiv: clinical case series," The Lancet HIV, 2020.

[7] M. M. Li, M. R. MacDonald, and C. M. Rice, "To translate, or not to translate: viral and host mrna regulation by interferon-stimulated genes," Trends in cell biology, vol. 25 , no. 6, pp. 320-329, 2015.

[8] R. S. Cvetkovic and K. L. Goa, "Lopinavir/ritonavir," Drugs, vol. 63, no. 8, pp. 769802, 2003.

[9] Y. M. Arabi, A. Y. Asiri, A. M. Assiri, H. A. A. Jokhdar, A. Alothman, H. H. Balkhy, S. AlJohani, S. Al Harbi, S. Kojan, M. Al Jeraisy et al., "Treatment of middle east respiratory syndrome with a combination of lopinavir/ritonavir and interferon-beta1b (miracle trial): statistical analysis plan for a recursive two-stage group sequential randomized controlled trial," Trials, vol. 21, no. 1, pp. 1-8, 2020. 
[10] C. Chu, V. Cheng, I. Hung, M. Wong, K. Chan, K. Chan, R. Kao, L. Poon, C. Wong, Y. Guan et al., "Role of lopinavir/ritonavir in the treatment of sars initial virological and clinical findings," Thorax, vol. 59, no. 3, pp. 252-256, 2004.

[11] G. Li and E. De Clercq, "Therapeutic options for the 2019 novel coronavirus (2019ncov)," Nature Reviews Drug Discovery, 2020.

[12] Y.-C. Chang, Y.-A. Tung, K.-H. Lee, T.-F. Chen, Y.-C. Hsiao, H.-C. Chang, T.-T. Hsieh, C.-H. Su, S.-S. Wang, J.-Y. Yu et al., "Potential therapeutic agents for covid-19 based on the analysis of protease and rna polymerase docking," Preprints, 2020, doi: 10.20944/preprints202002.0242.v2.

[13] L. Chen and G. Huang, "The antiviral activity of polysaccharides and their derivatives," International journal of biological macromolecules, vol. 115, pp. 77-82, 2018.

[14] C. Schaper, "Endogenous Binding of Steroid Molecules to DNA Nucleotides by a Ca2+/PO4- Process to Enable Gene Transcription," ChemRxiv, 2020, https://doi.org/10.26434/chemrxiv.11868261.v2.

[15] C. Schaper, "Structural Symmetry of DNA Nucleotides and Steroid Hormones," ChemRxiv, 2020, https://doi.org/10.26434/chemrxiv.11991567.v1.

[16] C. Schaper, "A Mechanism of Action for Hydroxychloroquine and Azithromycin to Inhibit Coronavirus Disease COVID-19," ChemRxiv, 2020, https://doi.org/10.26434/chemrxiv.12148914.v1.

[17] C. Schaper, "Competitive Inhibition of Cortisol by Prostaglandins at the Ligand Binding Domain of Glucocorticoid Receptors," bioRxiv, 2019, https://doi.org/10.1101/851501.

[18] X. Liu, Y. Wang, and E. A. Ortlund, "First high-resolution crystal structures of the glucocorticoid receptor ligand-binding domain-peroxisome proliferator-activated gamma coactivator 1-alpha complex with endogenous and synthetic glucocorticoids," Molecular Pharmacology, vol. 96, no. 4, pp. 408-417, 2019.

[19] T. A. Halgren, "Merck molecular force field . i. basis, form, scope, parameterization, and performance of MMFF94," Journal of computational chemistry, vol. 17, no. 5-6, pp. $490-519,1996$ 